\section{Developing future leaders}

\section{DIVERSITY IN HEALTHCARE: WHY REPRESENTATION MATTERS}

Alaa Abdelgabar, Shikila Edwards, Monique Wheatley. ST6 Anaesthestic Registrar North West Deanery UK, Medical Student Barts and The London School of Medicine and Dentistry

\subsection{6/leader-2020-FMLM.52}

Aim African-Caribbean Medical Mentors (ACMM) was founded in 2017 to develop future leaders in healthcare from an African-Caribbean background. We currently have a team of 15 members who provide outreach, mentoring and networking opportunities in order to positively influence outcomes for black students and doctors. The 2017 GMC Medical School Annual Report showed that only 3.3\% of all UK medical students were of an African Caribbean background. ACMM was founded to address this inequality by empowering black students through providing the skills necessary to succeed in a medical career. Our aim is to increase our contribution to the development of black doctors, in order to improve the diversity of leadership roles within healthcare.

Method We worked with schools and participation groups at universities, as well as utilising social media to enable our target audience to access our resources. Working alongside organisations such as the British Medical Association has provided us with a platform to reach doctors and medical students throughout the UK. Our evaluation demonstrates that black students who wish to study medicine benefit from free resources and guided support through the application process.

Results In the three years since African-Caribbean Medical Mentors was founded we have seen increased success in university applications: $80 \%$ of mentees have received medical and dental school offers. We have reached over 1500 students through outreach sessions and over 450 people through our events; we anticipate further increases. Effective promotion and a successful track-record has placed us in a position to support more individuals over time.

Conclusion Our social media presence and insight into healthcare allows us to promote diversity in medicine, as well as career progression amongst aspiring future leaders from African-Caribbean backgrounds. Representation is pivotal in the development of a more diverse healthcare workforce.

\section{Understanding leadership through research}

\section{ASSOCIATIONS OF FOUR NURSE STAFFING PRACTICES WITH HOSPITAL MORTALITY: KEY LESSONS FOR HOSPITAL MANAGERS AND LEADERS}

Christian M Rochefort, Marie-Ėve Beauchamp, Li-Anne Audet, Michal Abrahamowicz, Patricia Bourgault. School of Nursing, Faculty of Medicine and Health Sciences, Université de Sherbrooke, Sherbrooke, Québec, Canada, Research Center, Charles-Le Moyne-SaguenayLac-Saint-Jean sur les innovations en santé, Longueuil, Québec, Canada, Research Center, Centre hospitalier universitaire de Sherbrooke, Sherbrooke, Québec, Canada, Center for Outcomes Research and Evaluation, MGill University Health Centre, Montreal, Quebec, Canada, Department of Epidemiology, Biostatistics and Occupational Health, Faculty of Medicine, MGill University, Montreal, Quebec, Canada
Background Cross-sectional studies of hospital-level administrative data have suggested that four nurse staffing practices using adequate staffing levels, higher proportions of Registered Nurses (RNs) (skill mix), and more educated and experienced RNs - are each associated with reduced hospital mortality. To increase the validity of this evidence, patient-level longitudinal studies assessing the simultaneous associations of these staffing practices with mortality are required.

Methods A dynamic cohort of 146,349 adult medical, surgical, and intensive care patients admitted to a Canadian university health center was followed for seven years (2010-2017). We used a multivariable Cox proportional hazards model to estimate the associations between patients' time-varying cumulative exposure to measures of $\mathrm{RN}$ understaffing, skill mix, education and experience, each relative to nursing unit and shift means, and the hazard of in-hospital mortality, while adjusting for patient and nursing unit characteristics, and modeling the current nursing unit of hospitalization as a random effect.

Results Overall, 4,854 in-hospital deaths occurred during 3,478,603 patient-shifts of follow-up (13.95 deaths/10,000 patient-shifts). In multivariable analyses, every $5 \%$ increase in the cumulative proportion of understaffed shifts was associated with a $1.0 \%$ increase in mortality (HR: 1.010 ; 95\%CI: $1.002-1.017 ; \mathrm{p}=0.009)$. Moreover, every 5\% increase in the cumulative proportion of worked hours by baccalaureateprepared RNs was associated with a $2.0 \%$ reduction of mortality (HR: $0.980 ; 95 \% \mathrm{CI}: 0.965-0.995, \mathrm{p}=0.008)$. RN experience and skill mix were not significantly associated with mortality.

Conclusions Reducing the frequency of understaffed shifts and increasing the proportion of baccalaureate-prepared RNs are associated with reduced hospital mortality.

\section{Palliative medicine}

\section{DEVELOPING A PALLIATIVE CARE SERVICE AT NIGHTINGALE NORTH WEST}

Ben Anderosn. Nightingale North West, UK

\subsection{6/leader-2020-FMLM.54}

The Nightingale North West recruited members of the medical and nursing teams from many different backgrounds. Some had not worked in adult medicine before, while others had been out of work.

I perceived a lack of confidence in palliative care from discussions with nurses and doctors in the ward environment. This was clearly going to be a crucial part of working at the Nightingale. Practitioners' confidence develops when their skills are high, empowering them to seek advice, and is a useful measure in the development of a quality service. Nursing and medical staff at the Nightingale were surveyed. Confidence in palliative care was reported at $65 \%$, and only $38 \%$ for those with no prior experience.

Acting as a junior doctor representative on the palliative care management team helped address these issues. We laid on training sessions for staff, focussed on the logistics of palliative care at the Nightingale, alongside a separate consultant led session addressing identified learning needs. These sessions signposted to specialist advice and guidelines, so staff knew 
how to access additional expertise and support. The management team adapted national guidelines to take into account the resources available at the Nightingale. These guidelines were made available on the hospital intranet, and in a physical resource folder on the ward.

These interventions modestly improved confidence in palliative care from $65 \%$ to $68 \%$ over 3 months, but significantly improved for those with no previous experience, from $38 \%$ to $51 \%$. All training attendees reported feeling more confident. Those who received the most training saw confidence improve by $8 \%$, compared to $2 \%$ for those who received the least.

Working as a junior doctor leader ensured the palliative care management team were aware of and responded to day to day issues on the ward affecting patient care, helping make interventions to improve the service.

\section{Leading innovation and improvement}

\section{ESTABLISHING A STANDALONE MENTORSHIP SCHEME FOR NEWLY GRADUATED FYIIS DURING THE COVID-19 PANDEMIC: FEEDBACK AND EXPERIENCES}

Adam Pailing*, Huma Naqvi, Harriet Marsland. Sandwell and West Birmingham Hospitals NHS Trust, UK

\subsection{6/leader-2020-FMLM.55}

Aim A cross-site trust in Birmingham was tasked with setting up and providing a 6-week internship scheme for 24 newly graduated doctors from medical school as extra support in the midst of the COVID-19 pandemic, termed FYi1s.

Method A bespoke 1:1 buddy scheme between an FYi1 and current FY1 was opted for. Wherever possible, FYi1s were placed on the specialty and site they were starting on in August, and their buddy would be an FY1 currently working on that specialty. Feedback was obtained at the end of the internship. 16 of the 24 FYi1s responded to the feedback with a mixture of multiple choice and free text responses addressing communication, support, responsibility and the 1:1 buddy scheme.

Results Feedback from the internship was overwhelmingly positive and used to modify features of the full induction process in August, such as dedicated coffee meetings solely for new starters to share their experiences without senior presence.

The buddy system received excellent feedback and gave each FYi1 a personal support mechanism during their first weeks at the trust. Overcrowding on surgical wards was raised as an issue with a proportionally larger number of FYi1s starting on surgical rather than medical specialties volunteering to for the internship. Individual FYi1s would sometimes have to shadow different senior doctors each day if their buddy was taking annual leave or was working out-ofhours (eg. night shifts). Some FYi1s also did not manage to spend time on their August ward or specialty as that area remained a COVID zone throughout the whole of the internship.

Conclusions A comprehensive and successful mentorship scheme gives new employees invaluable experience and support to enable them to perform without limitation when they do begin work on their own in August.

\section{THE VALUE OF WRITTEN INFORMATION IN IMPROVING PATIENT EXPERIENCE}

T Crotty*, E Keane, T Moran, TP O'Dwyer, M Rafferty, SG Khoo. St. Vincents University Hospital, Nutley Lane, Dublin 4, IRE

\subsection{6/leader-2020-FMLM.56}

Flexible nasendoscopy (FNE) is a common procedure performed in ENT outpatient clinics. Although the majority of patients tolerate the procedure reasonably well, approximately $25 \%$ patients find the procedure distressing. Considering written information is cheap and widely accessible, we provided patients with an information leaflet prior to performing FNE and measured their satisfaction using a structured questionnaire.

Our results demonstrated a large improvement in patient satisfaction and understanding post-procedure. Additionally, patients found physicians who administered the information leaflet as having explained the procedure more comprehensively than without a questionnaire.

As healthcare professionals, we are committed to continually trying to improve patient experience. Providing leadership and innovation in healthcare can seem like a daunting task. However, small, simple changes can often result in large improvements in patient outcome. As leaders, we must increase awareness of the potential implications of small changes, such as written information on patient satisfaction.

\section{Quality Improvement, Leadership, Workforce Wellbeing}

\section{FATIGUE \& FACILITIES AT KETTERING GENERAL HOSPITAL THE IMPORTANCE OF HIGH-QUALITY REST TO MAXIMISE THE PERFORMANCE OF JUNIOR DOCTORS AND ENSURE PATIENT SAFETY}

Geeth Silva, Aiken Yam, Jessica Court, Rabia Imtiaz.

\subsection{6/leader-2020-FMLM.57}

Introduction Junior doctors are increasingly working in an overstretched NHS. In 2018 Kettering General Hospital (KGH) was awarded $£ 60,800$ of government funds to create high-quality rest facilities and improve junior doctor wellbeing.

Methods Through auditing doctors working at KGH, alongside consulting senior management, a project initiation document was constructed to spend this money. From November 2019 to June $2020 £ 46,275$ was spent on creating new rest facilities, including separate sleeping and working areas. Furthermore, the purchase of modern furniture and equipment met the functional needs of busy junior doctors. One month after the completion of the project, the team conducted a post-action review by reauditing how the changes impacted morale, wellbeing and quality of patient care.

Results Now, the majority of doctors are happy with the current rest areas on offer at $\mathrm{KGH}(60 \%)$, and a majority feel that they will use the on-call room area (63\%). Overall, the feedback from both interviews and the JDF was positive and, the renovation improved morale and wellbeing. There was an 